\title{
Anthocyanin metabolites in human urine and serum
}

\author{
Colin D. Kay ${ }^{1,2}$, G. Mazza ${ }^{1,2 \star}$, Bruce J. Holub ${ }^{2}$ and Jian Wang ${ }^{3}$ \\ ${ }^{1}$ Pacific Agri-Food Research Center, Agriculture and Agri-Food Canada, 4200 Hwy 97 Summerland, \\ British Columbia, Canada VOH 1 ZO \\ ${ }^{2}$ Department of Human Biology and Nutritional Sciences, University of Guelph, Ontario, Canada \\ ${ }^{3}$ Calgary Laboratory, Canadian Food Inspection Agency, Calgary, Alberta, Canada
}

(Received 26 September 2003 - Revised 2 February 2004 - Accepted 11 February 2004)

In the present study we investigated the metabolic conversion of cyanidin glycosides in human subjects using solid-phase extraction, HPLC-diode array detector, MS, GC, and enzymic techniques. Volunteers consumed approximately $20 \mathrm{~g}$ chokeberry extract containing $1.3 \mathrm{~g}$ cyanidin 3 -glycosides ( $899 \mathrm{mg}$ cyanidin 3 -galactoside, $321 \mathrm{mg}$ cyanidin 3 -arabinoside, $51 \mathrm{mg}$ cyanidin 3 -xyloside and $50 \mathrm{mg}$ cyanidin 3 -glucoside). Blood samples were drawn at $0,0 \cdot 5,1$, and $2 \mathrm{~h}$ post-consumption of the extract. Urine samples were also collected at $0,4-5$, and $22-24 \mathrm{~h}$. We have confirmed that human subjects have the capacity to metabolise cyanidin 3-glycosides, as we observed at least ten individual anthocyanin metabolites in the urine and serum. Average concentrations of anthocyanins and anthocyanin metabolites in the urine reached levels of 17.9 (range 14.9-20.9) $\mu \mathrm{mol} / 1$ within $5 \mathrm{~h}$ post-consumption and persisted in $24 \mathrm{~h}$ urine samples at levels of 12.1 (range 11.1-13.0) nmol/l. In addition, average total levels of anthocyanins and anthocyanin metabolites detected in the serum were observed at 591.7 (range 197.3-986.1) nmol/1 within $2 \mathrm{~h}$ post-consumption. Cyanidin 3-galactoside accounted for $55.4 \%$ (9.9 (range 7.2-12.6) $\mu \mathrm{mol} / \mathrm{l}$ ) and 66.0\% (390.6 (range 119.4-661.9) nmol/l) of the detected anthocyanins in the urine and serum samples, respectively. The metabolites were identified as glucuronide conjugates, as well as methylated and oxidised derivatives of cyanidin 3galactoside and cyanidin glucuronide. Conjugation probably affects the biological activity of anthocyanins and these metabolic products are likely in part responsible for the reported health benefits associated with the consumption of anthocyanins.

Flavonoids: Cyanidin 3-glycosides: Glucuronide: Methylated anthocyanins

In recent years, numerous studies have suggested that anthocyanins, present in fruit and vegetable products, are protective against many chronic degenerative diseases (Kamei et al. 1995; Laplaud et al. 1997; Andriambeloson et al. 1998; Trevithick \& Mitton, 1999; Mazza, 2000; Parthasarathy et al. 2001). However, there is little reliable information on their absorption and metabolism in human subjects. Several investigators report that anthocyanins are transported in biological fluids exclusively as intact glycosides (Miyazawa et al. 1999; Murkovic et al. 2000; Mazza et al. 2002; Mülleder et al. 2002; Suda et al. 2002), while few have identified glucuronide or sulfide derivatives (Wu et al. 2002; Felgines et al. 2003). An adequate identification of metabolised anthocyanins must be established before the elicitation of their health effects. Since conjugation and derivatisation probably alter the bioactive properties of anthocyanins, future in vitro studies should be conducted using anthocyanins in their metabolised forms, as they appear in the human body.

The aim of the present study was to investigate the metabolic fate of cyanidin 3-glycosides through the identification of intact or conjugated structures in human urine and serum.

\section{Experimental methods}

Material and reagents. The chokeberry extract (no. 74190000, lot L18010) was purchased from Artemis International, Inc. (Fort Wayne, IN, USA). The $\beta$-glucuronidase (type-3), $\quad \beta$-galactosidase (Aspergillus), $\quad \beta$-glucosidase (from almonds) and sulfatase (aryl sulfatase) were purchased from Sigma (Oakville, ON, Canada). The anthocyanin standards, cyanidin 3 -glucoside chloride, cyanidin 3-galactoside chloride (ideain chloride), malvidin 3-glucoside chloride (oenin chloride), peonidin 3-glucoside chloride, and pelargonidin 3-glucoside chloride (callistephin) were purchased from Extrasynthese (ZI Lyon Nord, Genay, France). The phenolic acid standards, syringic acid, vanillic acid, p-hydroxybenzoic acid, protocatechuic acid, caffeic acid, and gallic acid were purchased from Sigma (Oakville, ON, Canada). Ferulic acid was acquired from $\mathrm{K} \& \mathrm{~K}$ Rare \& Fine Chemicals (Costa Mesa, CA, USA) and p-hydroxycinnamic acid

\footnotetext{
Abbreviations: DAD, diode array detector; $\mathrm{E}_{440}: \mathrm{E}_{\mathrm{max}}$, absorbance intensity at $440 \mathrm{~nm} v$. maximum absorbance intensity; $\mathrm{m}+$, increased mass; MeOH, methanol; $m / z$, mass:charge ratio.

* Corresponding author: Dr G. (Joe) Mazza, fax +1 250494 0755, email mazzag@agr.gc.ca
} 
(or trans-p-hydroxycinnamic acid) was obtained from Baker Chemical Co. (Phillipsburg, NJ, USA). The Tri-Sil Z (Pierce, Rockford, IL, USA), methanol (MeOH), chloroform (Caledon, Toronto, ON, Canada), formic acid (Fisher Scientific, Springfield, NJ, USA), oxalic acid (Baker Chemical Co., Phillipsburg, NJ, USA), amyl alcohol, $\mathrm{HCl}$, and trifluoroacetic acid (DH Ltd, Toronto, ON, Canada) were all reagent grade. All solvents used for HPLC analysis were HPLC grade.

\section{Clinical procedures}

Two healthy, male volunteers, aged 27 and 54 years, participated in the chokeberry consumption trial. The subjects had an average BMI of $27.5 \mathrm{~kg} / \mathrm{m}^{2}$ and were absent of clinical disease as determined using a medical history questionnaire. The subjects were instructed to consume an essentially anthocyanin-free diet for $2 \mathrm{~d}$ before the study and to avoid taking aspirin or anti-inflammatory medications, and antioxidant or herbal supplements for 2 weeks before the investigation. The major constituents of the anthocyanin-free wash-out diet were milk, tuna, white bread, chicken, and white rice. No energy restrictions were imposed. The $2 \mathrm{~d}$ wash-out diet was performed before baseline sampling. After the overnight fast (12-14 h), the volunteers consumed approximately $20 \mathrm{~g}$ chokeberry extract (containing $1.3 \mathrm{~g}$ cyanidin glycosides) dissolved in $250 \mathrm{ml}$ water. The extract contained four cyanidin 3-glycosides (mg): cyanidin 3-galactoside, 899; cyanidin 3-arabinoside, 321; cyanidin 3-xyloside, 51; cyanidin 3-glucuoside, 50 (as determined by HPLC-diode array detector (DAD)). The chromatogram of the chokeberry extract is given in Fig. 1 (trace B). The extract was consumed directly following a baseline blood sample (time zero). Subsequent blood samples were taken at $0 \cdot 5,1$, and $2 \mathrm{~h}$ post-consumption of the extract. Blood samples were drawn by venepuncture from a brachial vein into $10 \mathrm{ml}$ evacuated glass tubes (Vacutainer; Becton Dickinson, Franklin Lakes, NJ, USA). The blood samples were allowed to clot at room temperature for $25 \mathrm{~min}$. Samples were then immediately centrifuged $(2500 \mathrm{rpm}, 1000 \mathrm{~g})$ for $15 \mathrm{~min}$ at $5^{\circ} \mathrm{C}$ to recover the serum. Urine voids were collected in the morning of the study date (first void, time zero) along with $4-5$ and $22-24 \mathrm{~h}$ samples. The serum and urine were stored at $-70^{\circ} \mathrm{C}$ upon removal or collection.

\section{Anthocyanin extraction}

Anthocyanins were extracted from biological fluids using disposable solid-phase extraction $\mathrm{C}_{18}$ cartridges (Supelclean ENVI-18 $6 \mathrm{ml} 500 \mathrm{mg}$; Sigma, Oakville, ON, Canada). Cartridges were pre-conditioned using $7 \mathrm{ml}$ acidified $\mathrm{MeOH}(0.1 \%$ trifluoroacetic acid, $\mathrm{pH} 2 \cdot 1)$, followed by $7 \mathrm{ml}$ acidified water (10 mM-oxalic acid, $\mathrm{pH} 2 \cdot 2)$. Unfiltered blood serum $(1.5 \mathrm{ml})$ or unfiltered urine $(1 \mathrm{ml})$ was acidified $(6 \mathrm{M}-\mathrm{HCl} ; 40 \mu \mathrm{l})$, diluted with $10 \mathrm{mM}$-oxalic acid (equal volume), vortexed, and loaded directly onto the solid-phase extraction cartridge. The sample was drained under gravity (about $1 \mathrm{drop} / \mathrm{s}$ ) and washed with 2 volumes of acidified water (10 mM-oxalic acid, approximately $12 \mathrm{ml}$ ). The remaining anthocyanin extract was eluted with $6 \mathrm{ml}$ acidified $\mathrm{MeOH}(0.1 \%$ trifluoroacetic acid). The extract was evaporated at ambient temperature in a SpeedVac Plus-SC110A condenser (Savant Instruments Inc., Farmingdale, NY, USA) to $0.1-0.5 \mathrm{ml}$. The residual elute was brought to dryness under $\mathrm{N}_{2}$ at room temperature and the residue was re-dissolved in $200 \mu \mathrm{l}$ HPLC mobile-phase solution and filtered through a $13 \mathrm{~mm} 0.45 \mu \mathrm{m}$ GHB Acrodisc syringe filter (lot no. A10310523; Pall-Gelman, Ann Arbor, MI, USA). Anthocyanins were quantified by comparison with a standard curve obtained using known concentrations of cyanidin 3-galactoside.

\section{High-pressure liquid chromatography analysis}

HPLC analysis was performed on an Agilent 1100 series HPLC (Agilent Technologies, Palo Alto, CA, USA) using a Zorbax SB $\mathrm{C}_{18}(5 \mu \mathrm{m}, 4.6 \times 250 \mathrm{~mm}$; Agilent Technologies, Palo Alto, CA, USA) reverse-phase column with an Inertsil Spherisorb guard column $\left(\mathrm{C}_{18} 5 \mu \mathrm{m}, 4.6 \times 30 \mathrm{~mm}\right.$; Phenomenex, Torrance, CA, USA). The column temperature was set at $35^{\circ} \mathrm{C}$ and the injector temperature at $15^{\circ} \mathrm{C}$, with an injection volume of $50 \mu \mathrm{l}$. The mobile phase consisted of $4.5 \%(\mathrm{v} / \mathrm{v})$ formic acid in water (solvent A) and $100 \% \mathrm{MeOH}$ (solvent B). The flow rate was $1.0 \mathrm{ml} /$ min and the solvent gradient program used as follows: $10 \% \mathrm{~B}$ at $0 \mathrm{~min}, 25 \% \mathrm{~B}$ at $30 \mathrm{~min}, 45 \% \mathrm{~B}$ at $50 \mathrm{~min}$, $100 \% \mathrm{~B}$ at $55 \mathrm{~min}$, maintaining $100 \%$ for $10 \mathrm{~min}$. A DAD (G1315B; Agilent Technologies, Palo Alto, CA, USA) monitored absorbance at 280, 360, and $525 \mathrm{~nm}$. Peaks on the chromatogram corresponding to anthocyanins as identified by spectral analysis (peaks detected at $525 \mathrm{~nm}$ with $\lambda_{\max }$ 250-300, 500-550) were collected from the analytical column (Foxy $200 \mathrm{X}-\mathrm{Y}$ fraction collector; ISCO, Inc., Lincoln, NE, USA) and concentrated at ambient temperature in a vacuum condenser (SpeedVac; Savant, Farmingdale, NY, USA) for further analysis. All water used for HPLC was $18 \mathrm{M} \Omega \times \mathrm{cm}$ Milli-Q water and degassed using an inline Agilent 1100 micro vacuum degasser (Agilent Technologies, Palo Alto, CA, USA).

\section{Mass spectrometry analysis}

MS identification of individual compounds was conducted post-separation via HPLC (as described above). Individual peaks were collected on a Foxy 200 X-Y fraction collector (ISCO, Inc., Lincoln, NE, USA), concentrated, and analysed by electrospray ionisation-MS. A Micromass ZQ single quadrupole mass spectrometer with electrospray interface and MassLynx 3.5 software (Micromass UK Ltd, Manchester, UK) was used for data acquisition. The mass spectrometer parameters settings were: ionisation mode, electrospray positive ion mode; capillary voltage, $3.25 \mathrm{kV}$; source temperature, $130^{\circ} \mathrm{C}$; desolvation temperature, $280^{\circ} \mathrm{C}$; nebuliser $\mathrm{N}_{2}$ flow rate, 95 litres/h; desolvation $\mathrm{N}_{2}$ gas flow rate, 610 litres/h; LM resolution, 15; HM resolution, 15; ion energy, $0.8 \mathrm{~V}$; multiplier voltage, $650 \mathrm{~V}$; cone voltage, $20 \mathrm{~V}$; RF lens, $0.5 \mathrm{~V}$; extractor, $6 \mathrm{~V}$. The flow rate of a built-in syringe pump was set at $20 \mu \mathrm{l} / \mathrm{min}$. For the flow injection, samples (HPLC fractions) and chokeberry extracts $(2.4 \mathrm{mg})$ were 


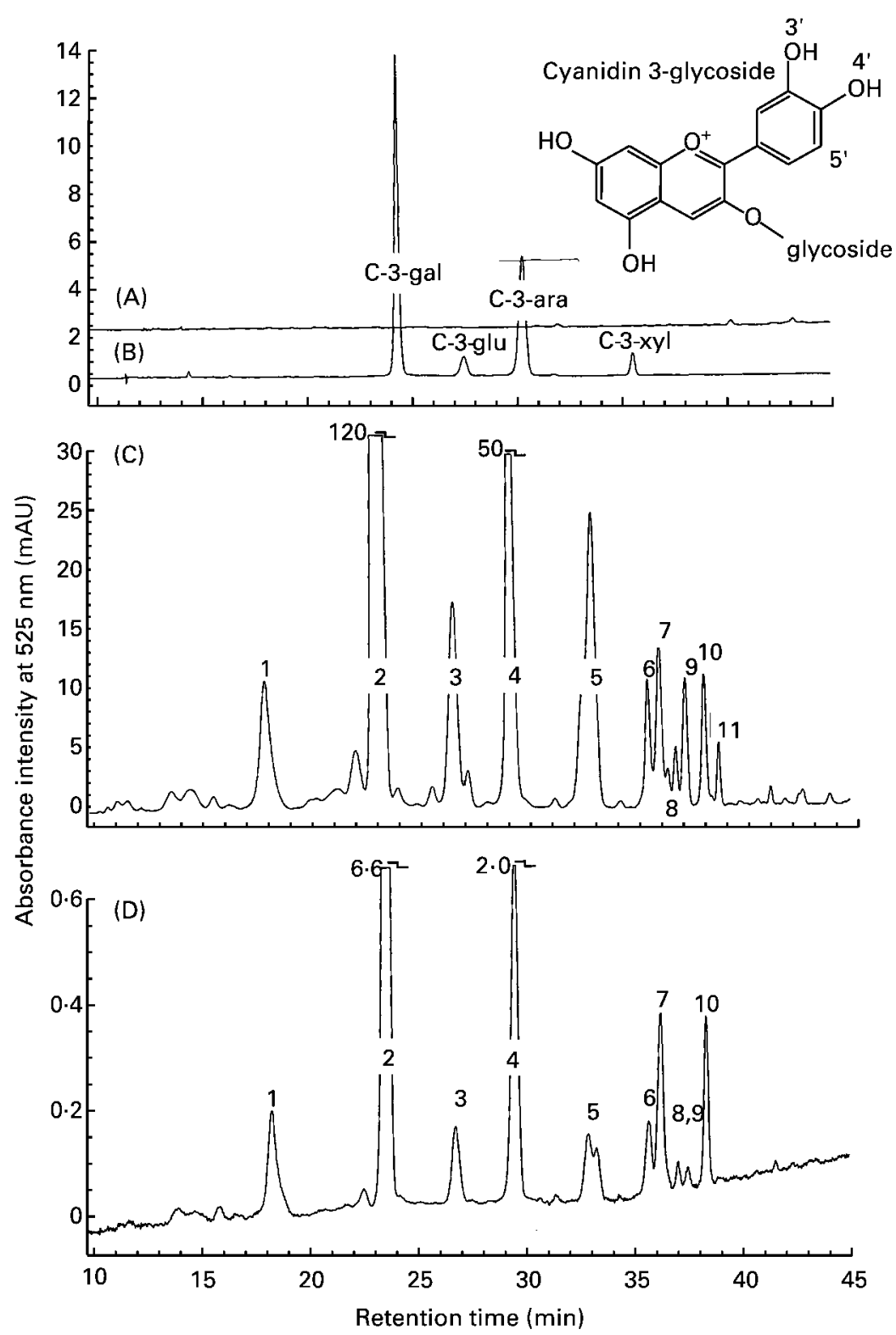

Fig. 1. Anthocyanins in chokeberry extract, and human urine and serum post-consumption of extract. (A), Chromatogram of baseline urine sample; (B), chromatogram of chokeberry extract; (C), chromatogram of $5 \mathrm{~h}$ urine sample; (D), chromatogram of $2 \mathrm{~h}$ serum sample; C-3-gal, cyanidin 3-galactoside; C-3-glu, cyanidin 3-glucoside; C-3-ara, cyanidin 3-arabinoside; C-3-xyl, cyanidin 3-xyloside. HPLC analysis was as outlined on p. 934. Identification of chokeberry anthocyanins was based on that of known standards. When standards were not available identification was based on retention times and spectral data from the literature (Chandra et al. 2001). Urine and serum concentrations of each compound represented by peaks 1 to 11 are given in Tables 1 and 2, respectively. Urine and serum concentrations of anthocyanins were determined using UV-visible HPLC and quantified in relation to known concentrations of a C-3-gal standard.

re-dissolved in $2 \mathrm{ml}$ acetonitrile-water (50:50) containing $0.1 \%$ formic acid. Spectra were recorded by scanning a mass range from mass:charge ratio $(\mathrm{m} / \mathrm{z}) 100$ to 1000 with a scan time of $1 \mathrm{~s}$, an inter-scan time of $0.02 \mathrm{~s}$, and a run duration of $0.5 \mathrm{~min}$.

\section{Enzymic hydrolysis}

The dried anthocyanin extract obtained using the solidphase extraction procedure outlined earlier (p. 934) was subjected to enzymic hydrolysis using $\beta$-galactosidase, $\beta$-glucuronidase, $\beta$-glucosidase, and sulfatase. The dried extract was mixed with $0 \cdot 1$ M-sodium acetate buffer
( $1 \mathrm{ml}$ ) containing activated enzyme. The four individual enzyme buffer solutions (700 U $\beta$-galactosidase, $500 \mathrm{U}$ $\beta$-glucuronidase, $500 \mathrm{U} \beta$-glucosidase, $75 \mathrm{U}$ sulfatase) were created by adding $1 \mathrm{ml}$ pre-incubated $\left(37^{\circ} \mathrm{C}\right)$ sodium acetate buffer $(\mathrm{pH} \mathrm{3.8)}$ to the pre-weighed enzymes. The enzyme buffer solutions were then vortexed and added to the dried anthocyanin extract. The mixtures were further vortexed and incubated at $37^{\circ} \mathrm{C}$ for $1 \mathrm{~h}$. The anthocyanin enzyme extracts were then diluted with $1 \mathrm{ml}$ acidified $\mathrm{MeOH}(4.5 \%$ formic acid in $\mathrm{MeOH}, \mathrm{pH} 2.1)$ and centrifuged for $10 \mathrm{~min}$ at $14000 \mathrm{rpm}$. The supernatant fractions were removed, evaporated under $\mathrm{N}_{2}$ and re-dissolved in $150 \mu \mathrm{l}$ mobile phase (as outlined earlier; 
p. 934). If precipitate persisted, the sample was re-centrifuged (10 min at $14000 \mathrm{rpm})$ before filtration. The enzymically hydrolysed aglycone-rich extracts were injected into the HPLC column and analysed using the HPLC methodology outlined earlier (p. 934).

Gas chromatography analysis, acid hydrolysis and derivatisation of sugars

Chemical characterisation of the glycosylating compounds was conducted using capillary GLC post-acid hydrolysis. This was accomplished using methods as published by Gao \& Mazza (1994), with slight modifications. Samples were silyated after concentration by adding $50 \mu \mathrm{l}$ Tri-Sil $\mathrm{Z}$, and incubated at $65^{\circ} \mathrm{C}$ for $1 \mathrm{~h}$. GC analysis of the derivatised sugars was conducted on a Hewlett Packard 5890A gas chromatograph with a flame ionisation detector (Hewlett Packard (now Agilent Technologies), Palo Alto, CA, USA) using a fused silica capillary column (J\&W DB-1701, $30 \mathrm{~m} \times 0.32 \mathrm{~mm} \times 1 \mu \mathrm{m}$; J\&W Scientific Inc., Folsom, CA, USA). The injector and detector temperatures were $250^{\circ} \mathrm{C}$. The carrier gas was He at a head pressure of $80 \mathrm{kPa}$, flow of $1.8 \mathrm{ml} / \mathrm{min}$, and a linear velocity of $350 \mathrm{~mm} / \mathrm{s}$. The sample $(1 \mu \mathrm{l})$ was injected into the column via a split/splitless injector in split mode using a split ratio of $11: 1$. The initial column temperature was increased from 120 to $180^{\circ} \mathrm{C}$ at a rate of $20^{\circ} \mathrm{C} / \mathrm{min}$, then increased from 180 to $200^{\circ} \mathrm{C}$ at a rate of $5^{\circ} \mathrm{C} / \mathrm{min}$ and held for $8 \mathrm{~min}$.

\section{Results}

The consumption of chokeberry extract containing four cyanidin glycosides $(899 \mathrm{mg}$ cyanidin 3-galactoside, $321 \mathrm{mg}$ cyanidin 3-arabinoside, $51 \mathrm{mg}$ cyanidin 3-xyloside, and $50 \mathrm{mg}$ cyanidin 3-glucuoside) resulted in the appearance of at least ten anthocyanin metabolites (as separated by reverse-phase HPLC-DAD) in the human serum and urine (Figs. 1 and 2). Concentrations of individual anthocyanins in the urine and serum extracts are given in Tables 1 and 2 respectively. HPLC-DAD analysis of anthocyanin standards together with their molecular
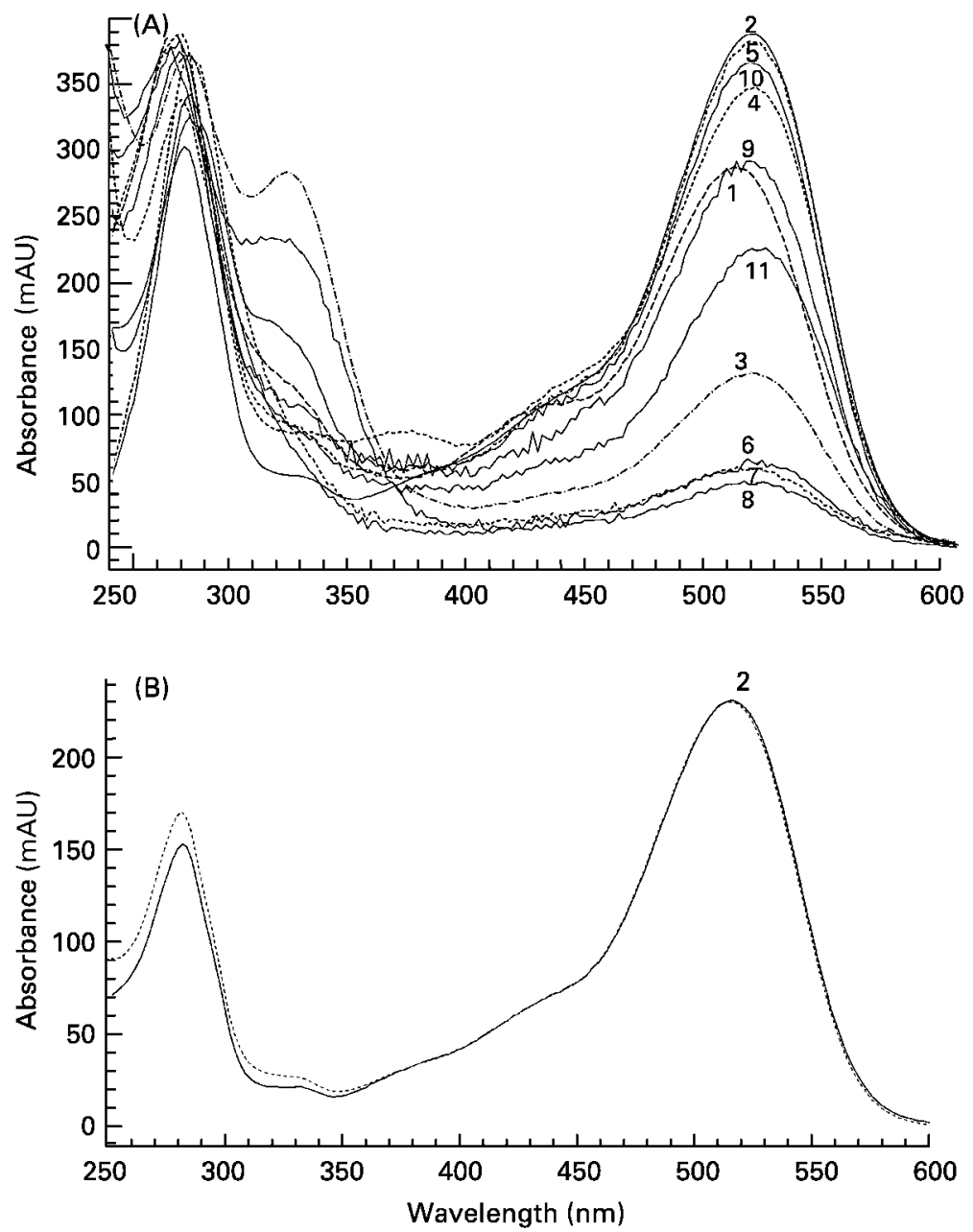

Fig. 2. UV-visible absorption spectra of anthocyanins in human urine and serum samples post-consumption of chokeberry extract monitored at $525 \mathrm{~nm}$ by HPLC-diode array detector (DAD). (A), Typical UV-visible absorption spectra of individual peaks in human urine and serum samples post-consumption of the extract; (B), UV-visible absorption spectra of peak 2 ( - ) and cyanidin 3-galactoside standard (- - -). HPLC chromatogram of urine and serum samples are given in Fig. 1. Urine and serum concentrations of each compound represented by peaks 1 to 11 are given in Tables 1 and 2, respectively. Urine and serum concentrations of anthocyanins were determined using HPLC-DAD and quantified in relation to known concentrations of a cyanidin 3-galactoside standard. 
Table 1. Identification and concentration of anthocyanins and anthocyanin metabolites in human urine

\begin{tabular}{|c|c|c|c|c|c|c|c|c|}
\hline \multirow[b]{2}{*}{ Peak } & \multirow[b]{2}{*}{$\mathrm{Rt}^{\star}(\min )$} & \multirow[b]{2}{*}{$\lambda_{\max }{ }^{*}(\mathrm{~nm})$} & \multirow[b]{2}{*}{$\mathrm{E}_{440}: \mathrm{E}_{\max }{ }^{*}($ as $\%)$} & \multirow{2}{*}{$\begin{array}{c}\mathrm{m} / \mathrm{z \dagger} \\
\text { (total/aglycone) }\end{array}$} & \multirow{2}{*}{$\begin{array}{c}\text { Conjugation and } \\
\text { derivatization }\end{array}$} & \multicolumn{3}{|c|}{ Concentration $(\mu \mathrm{mol} / \mathrm{l}) \S$} \\
\hline & & & & & & Subject 1 & Subject 2 & Average \\
\hline 1 & $18 \cdot 1$ & 510 & 39 & 449 & Unknown & 0.5 & 0.7 & 0.6 \\
\hline 2 & $23 \cdot 4$ & 515 & 31 & 449 & Intact C-3-gal & $12 \cdot 6$ & $7 \cdot 2$ & 9.9 \\
\hline 3 & 26.5 & 516 & 24 & 463 & Gluc & 0.7 & 0.9 & 0.8 \\
\hline 4 & $29 \cdot 3$ & 517 & 37 & $463 / 301$ & $\mathrm{CH}_{3}$ & $3 \cdot 3$ & $2 \cdot 2$ & $2 \cdot 7$ \\
\hline 5 & 32.9 & 515 & 36 & $463 / 301$ & $\mathrm{CH}_{3}$ & $2 \cdot 1$ & 1.6 & 1.8 \\
\hline 6 & 35.5 & 516 & 33 & 447 & Gluc $+\mathrm{CH}_{3}$ & 0.2 & 0.5 & 0.3 \\
\hline 7 & $36 \cdot 0$ & 514 & 40 & 447 & Gluc $+\mathrm{CH}_{3}$ & 0.1 & 0.7 & 0.4 \\
\hline 8 & $36 \cdot 8$ & 516 & 38 & Unknown & Unknown & 0.3 & 0.3 & 0.3 \\
\hline 9 & $37 \cdot 7$ & 517 & 30 & 447 & $2 \times \mathrm{CH}_{3}$ & 0.6 & 0.4 & 0.5 \\
\hline 10 & 38.5 & 520 & 30 & 491 & Gluc $+2 \times \mathrm{CH}_{3}$ & 0.5 & 0.4 & 0.4 \\
\hline 11 & $39 \cdot 0$ & 518 & 31 & $493 / 331$ & $2 \times \mathrm{CH}_{3}+\mathrm{OH}$ & 0.1 & 0.1 & 0.1 \\
\hline Total & & & & & & $20 \cdot 9$ & 14.9 & $17 \cdot 9$ \\
\hline
\end{tabular}

Rt, retention time; $\mathrm{E}_{440}: \mathrm{E}_{\max }$, absorbance intensity at $440 \mathrm{~nm} v$. maximum absorbance intensity; m/z, mass:charge; C-3-gal, cyanidin 3-galactoside; Gluc, glucuronide acid.

${ }^{*} \mathrm{Rt}$ and spectral data were obtained by HPLC as outlined on p. 934. For an HPLC chromatographic plot of individual peaks, see Fig. 1.

† Data were obtained by MS. Anthocyanins were ionized in their molecular cation forms under MS positive ion mode (as outlined on p. 934).

¥Identification was based on relative polarity (Rt), MS, and spectral data (as outlined on p. 934) using standards of known composition when available. For spectral characteristics of anthocyanin standards, see Table 3.

$\S$ Concentrations ( $\mu \mathrm{mol} / \mathrm{l}) 5 \mathrm{~h}$ following consumption of two subjects determined using UV-visible HPLC and quantified in relation to known concentrations of a C-3-gal standard. No anthocyanins were detected in baseline samples.

Table 2. Concentrations of identifiable anthocyanins in human serum*

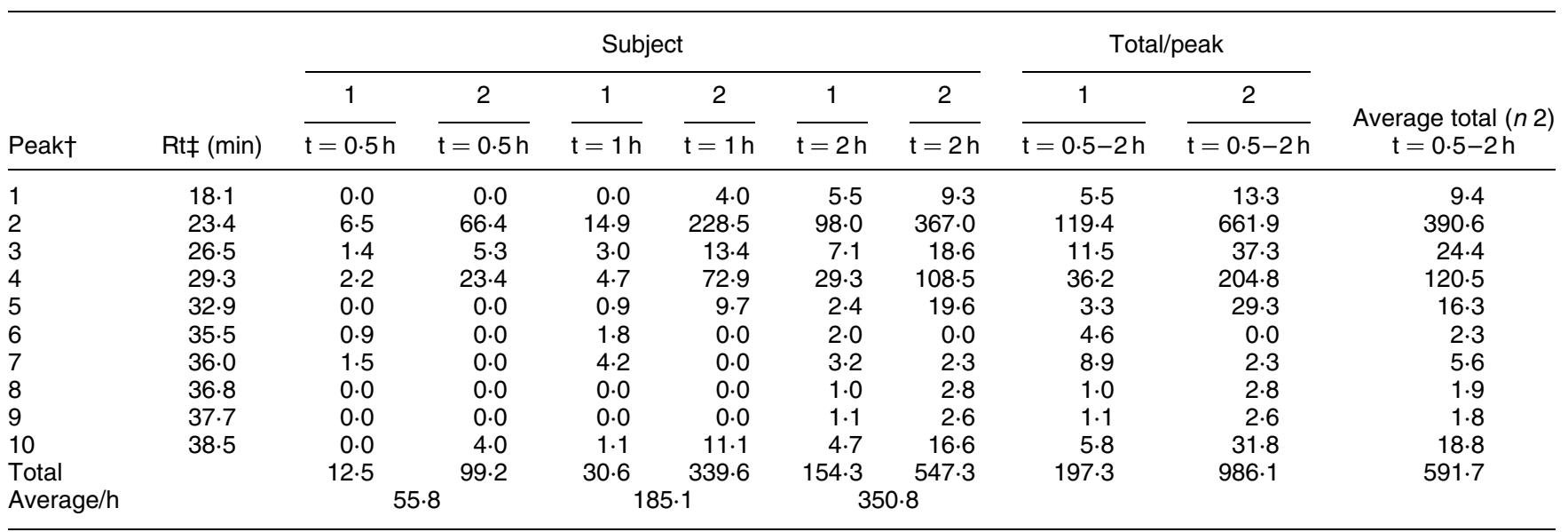

${ }^{*}$ Concentrations $(\mathrm{nmol} / \mathrm{l})$ of two subjects were determined using UV-visible HPLC and quantified in relation to known concentrations of a cyanidin 3-galactoside standard. For an HPLC chromatographic plot of individual peaks, see Fig. 1; for MS and HPLC identification of individual peaks, see Table 1. No anthocyanins were detected in baseline samples.

†Individual peaks were identified as anthocyanins by analysis of UV-visible spectra having maxima in the $250-300$ and $500-550 \mathrm{~nm}$ range.

$\ddagger$ Retention times were obtained by HPLC as outlined on p. 934

weights are given in Table 3. The UV-visible spectral analysis of all potential metabolites is given in Fig. 2. Initial attempts to fully characterise anthocyanin metabolites in the urine by enzymic and chromatographic methods were unsuccessful and results of both enzymic hydrolysis (detection of aglycone via HPLC-DAD) and acid hydrolysis (detection of derivatised sugars and glucuronic acid via capillary GC) experiments were deemed inconclusive. MS was necessary for the adequate identification of urinary metabolites. Post-MS analysis, the identification of anthocyanins (Table 1) was based on the matching of molecular weights for parent (anthocyanin) and daughter (anthocyanidin or aglycone; when obtainable) fragments, along with HPLC-DAD data (retention time, $\lambda_{\max }$ (vis) and absorbance intensity at $440 \mathrm{~nm} v$. maximum absorbance intensity $\left.\left(\mathrm{E}_{440}: \mathrm{E}_{\max }\right)\right)$ with that of available standards
(Table 3). Identification was based on metabolites isolated from pooled urine samples. The concentration of unidentified metabolites is based on cyanidin 3-galactoside molar equivalents. The results indicated that cyanidin 3-galactoside was the primary anthocyanin in the chokeberry extract accounting for $68.0 \%$ of its total anthocyanins. Cyanidin 3galactoside was also the primary anthocyanin identified in the urine and serum samples accounting for $55.3 \%$ (9.9 (range $7.2-12.6) \mu \mathrm{mol} / \mathrm{l}$ ) and 66.0\% (390.6 (range 119.4-661.9) $\mu \mathrm{mol} / \mathrm{l}$ ) of the identified anthocyanins, respectively. No anthocyanins were detected in the baseline serum or urine samples. The results indicated the presence of both cyanidin 3-galactoside $(\mathrm{m} / \mathrm{z} 449$; Fig. 1, peak 2) and cyanidin glucuronide $(\mathrm{m} / \mathrm{z}$ 463; Fig. 1, peak $3)$. The results also indicated the presence of mono- and dimethylated cyanidin galactosides (increased mass 
Table 3. Ultraviolet-visible high-pressure liquid chromatography characteristics of anthocyanin standards

\begin{tabular}{|c|c|c|c|c|c|}
\hline \multirow[b]{2}{*}{ Peak } & \multirow[b]{2}{*}{$\mathrm{Rt}^{\star}(\min )$} & \multicolumn{2}{|c|}{ Absorption spectra } & \multicolumn{2}{|c|}{ Molecular weight $(\mathrm{m} / \mathrm{z})$} \\
\hline & & $\lambda_{\max (\text { vis) }}(\mathrm{nm})$ & $\mathrm{E}_{440}: \mathrm{E}_{\max }(\mathrm{as} \%)$ & Glycoside & Aglycone \\
\hline Cyanidin-3-galactoside & $24 \cdot 1$ & 518 & $31 \cdot 7$ & 449 & 287 \\
\hline Cyanidin-3-glucoside & $26 \cdot 6$ & 517 & $32 \cdot 5$ & 449 & 287 \\
\hline Cyanidin-3-arabinoside $†$ & $29 \cdot 2$ & 517 & $30 \cdot 7$ & 419 & 287 \\
\hline Pelargonidin-3-glucoside & $32 \cdot 3$ & 502 & $44 \cdot 0$ & 433 & 271 \\
\hline Peonidin-3-glucoside & $36 \cdot 0$ & 518 & $32 \cdot 6$ & 463 & 301 \\
\hline Malvidin-3-glucoside & 38.5 & 528 & 27.5 & 493 & 331 \\
\hline Cyanidin-3-xyloside† & 38.9 & 517 & $43 \cdot 6$ & 419 & 287 \\
\hline Cyanidin & $43 \cdot 0$ & 526 & $23 \cdot 5$ & & 287 \\
\hline
\end{tabular}

$R t$, retention time; $E_{440}: E_{\max }$, absorbance intensity at $440 \mathrm{~nm} v$. maximum absorbance intensity; $\mathrm{m} / \mathrm{z}$, mass:charge ratio.

${ }^{*}$ Rt and spectral data were obtained by HPLC (as outlined on p. 934) using standards of known composition when available.

†Identification of cyanidin 3-arabinoside and cyanidin 3-xyloside in the chokeberry extract was based on retention times and spectral data (as standards were not available) and was confirmed in the literature (Chandra et al. 2001)

$(\mathrm{m}+) 14$ and $\mathrm{m}+28$; Fig. 1 , peaks 4 and 5 respectively) and glucuronides $(\mathrm{m}+14$; Fig. 1 , peaks 6 and 7 and $\mathrm{m}+28$; Fig. 1, peak 10). Oxidative modification was also indicated in one peak $(\mathrm{m} / \mathrm{z}, 493 / 331$; Fig. 1, peak 11) by an increased mass $(\mathrm{m}+16)$ above the di-methyl derivative of cyanidin 3-galactoside (represented by $\mathrm{m} / \mathrm{z}$ 477, Fig. 1, peak 9). A proposed pathway for these compounds is given in Fig. 3.

The HPLC quantitative analysis of the individual anthocyanin peaks in the urine revealed that cyanidin 3-galactoside and derivatives of cyanidin 3-galactoside accounted for an average of $84.0 \%$ (15.0 (range 11.518.7) $\mu \mathrm{mol} / \mathrm{l}$ ) of the identified metabolites. Cyanidin glucuronide and glucuronide derivatives accounted for $10.6 \%(1.9$ (range $1.5-2.4) \mu \mathrm{mol} / \mathrm{l}$ ), with the remaining $5.0 \%$ or 0.9 (range $0.8-1.0$ ) $\mu \mathrm{mol} / 1$ uncharacterised (Fig. 1, traces $\mathrm{C}$ and $\mathrm{D}$, peaks 1 and 8 ). In addition to the urinary analysis of metabolites, comparisons were made between urinary and serum metabolites using HPLC-DAD data (Figs. 1 and 2, and Tables 1 and 2).

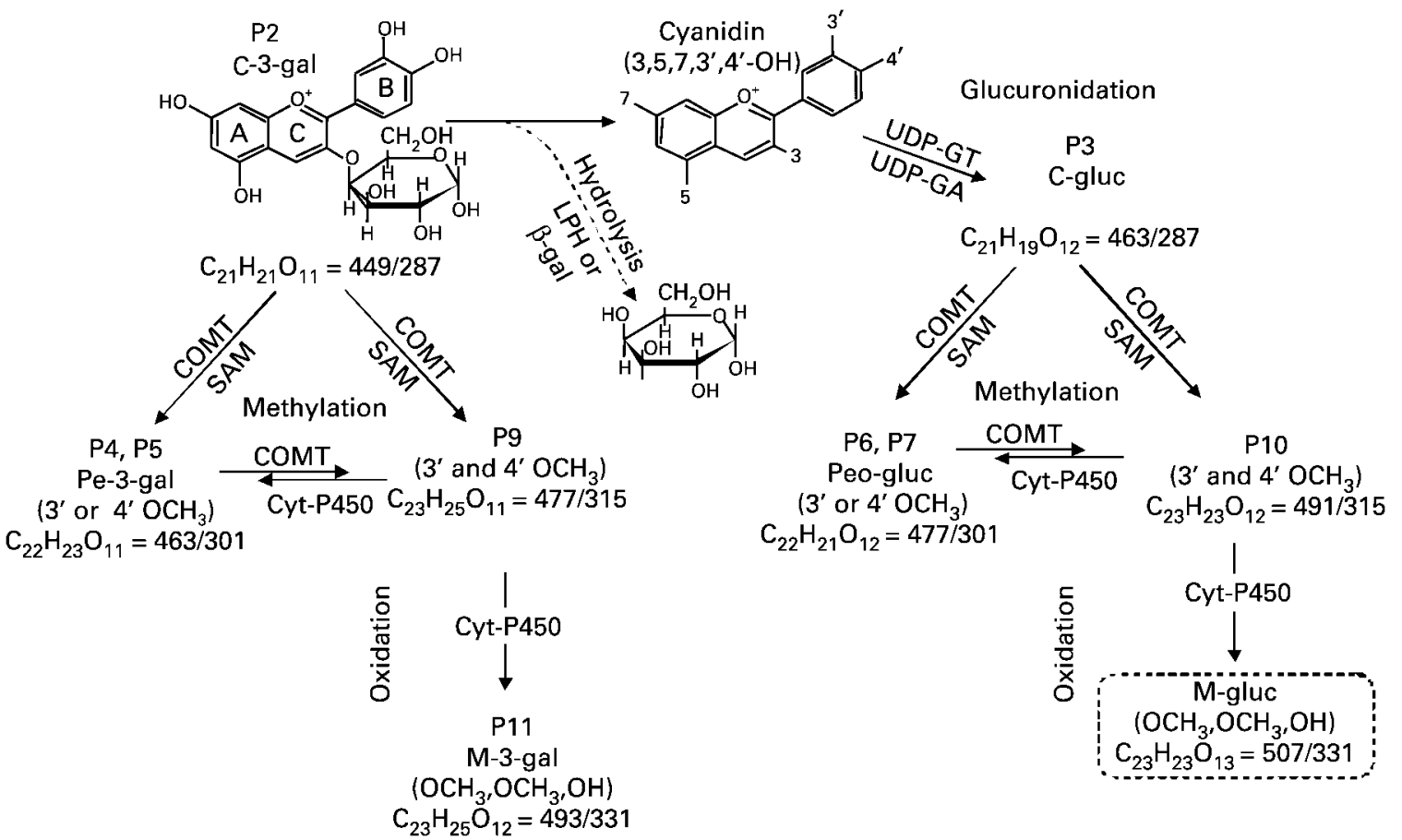

Fig. 3. Proposed pathway for the formation of anthocyanin metabolites in human urine and serum post-consumption of cyanidin 3-glycosides. $\mathrm{P}$, peak; C-3-gal, cyanidin 3-galactoside; C-gluc, cyanidin glucuronide; M-3-gal, malvidin 3-galactoside; M-gluc, malvidin glucuronide; Peo-3-gal, peonidin 3-galactoside; Peo-gluc, peonidin glucuronide; COMT, catechol-O-methyltransferase; SAM, S-adenosyl methionine; LPH, lactase-phlorizin hydrolase; $\beta$-gal, $\beta$-galactosidase; UDP-GT, uridine diphosphate glucuronosyltransferase; UDP-GA, UDP-glucuronic acid; Cyt-P450, cyotchrome P450. Molecular weights were obtained by MS (as outlined on p. 934). Identification was based on relative polarity (retention time), MS, and UV-visible data (as outlined on p. 934 and Table 1) and compared with those of known standards (Table 3). For an HPLC chromatographic plot of individual peaks, see Fig. 1. The circled metabolite is proposed but not identified. P1 and P8 are not illustrated as there were insufficient data available to elicit a potential structure. P9 and P10 have not been named as no anthocyanins of comparable structure have been reported to date. 
The results indicated that the serum peaks matched (both retention times and UV-visible spectra) the peaks identified in the urine with the exception of one compound (peak 11) that was observed in the urine but did not appear in the serum (Fig. 1, traces C and D). Cyanidin 3-galactoside and derivatives of cyanidin galactoside identified in the serum accounted for $89.4 \%$ (529.3 (range 160.0-898.6) $\mathrm{nmol} / \mathrm{l}$ ) of the metabolites. Glucuronide and glucuronide derivatives accounted for 8.6\% (51.1 (range 30.8-71.4) $\mathrm{nmol} / \mathrm{l}$ ), with the remaining $1.9 \%$ or 11.3 (range $6.5-$ 16.1) nmol/l uncharacterised (Fig. 1, traces $\mathrm{C}$ and $\mathrm{D}$, peaks 1 and 8 ).

\section{Discussion}

The purpose of the present study was to identify potential metabolites of cyanidin 3-glycosides in human urine and serum. To date there is little reliable information on the absorption and metabolism of anthocyanins in human subjects, and the studies available have reported contradictory results. The results of the present investigation indicate that a large proportion of anthocyanins consumed are metabolised before entry into the circulation and their metabolites will probably be responsible for many of the reported health effects associated with anthocyanin consumption.

The chokeberry extract utilised in the present trial was chosen because it contained only one anthocyanidin species, cyanidin. Most other fruit extracts contain two or more anthocyanidins making it difficult to identify the origin of any one metabolic by-product. Unfortunately, the highly concentrated chokeberry extract contained a high percentage of phenolics and polyphenolics. As a result, it was very astringent and its palatability (when dispersed in water) was low. Consequently, incomplete subject compliance made it difficult to establish the exact concentration of the supplement consumed by the two subjects. For this reason, no attempt has been made to establish the percentage of anthocyanins appearing in the urine and serum relative to the initial dose (bioavailability). Future studies will establish bioavailability using an encapsulated extract or a product more similar in composition to the intact fruit. The aim of the present study was simply to consume a high-enough concentration of cyanidin glycosides to identify their possible metabolites in biological fluids and to elicit a potential metabolic pathway for anthocyanin biotransformation in human subjects. It is important to note that the structural derivatives of anthocyanins proposed in the present study result from the consumption of a high dose of anthocyanins $(1.3 \mathrm{~g})$. The metabolic route under these circumstances may differ from the route following the ingestion of a more 'typical' dose of anthocyanins, as would be encountered with high fruit and vegetable consumption or with moderate to high wine consumption.

The consumption of four cyanidin glycosides (cyanidin 3-galactoside, cyanidin 3-arabinoside, cyanidin 3-xyloside, and cyanidin 3-glucoside) resulted in the appearance of at least ten individual anthocyanin metabolites in the human urine and serum (Figs. 1 and 2). To our knowledge, no other study has identified this number of anthocyanin metabolites in the urine or serum to date. Findings from studies using similar concentrations of total anthocyanins but reporting fewer metabolites are probably the result of individual metabolite concentrations being below the detection limit of the methodologies used. In the present study, only cyanidin was consumed, with the majority $(68 \%)$ attributed to one cyanidin glycoside (cyanidin 3galactoside), therefore, resulting in fewer numbers and higher concentrations of individual metabolites. In the present investigation, the total concentration of identifiable anthocyanins in the urine reached an average of 17.9 (range 14.9-20.9) $\mu \mathrm{mol} / \mathrm{l}$ within $5 \mathrm{~h}$ post-consumption of the chokeberry extract (Table 1). Urine samples (22$24 \mathrm{~h}$ ) showed cyanidin 3-galactoside and metabolised derivatives of cyanidin 3-galactoside to persist in the urine at levels of $0 \cdot 011-0 \cdot 013 \mathrm{nmol} / \mathrm{l}$. The identification of anthocyanin metabolites in $24 \mathrm{~h}$ urine samples has also recently been noted by Felgines et al. (2003), and may signify the potential for minor tissue accumulation. Additionally, the concentrations of identifiable anthocyanins and anthocyanin metabolites in the serum $(2 \mathrm{~h}$ sample) were observed at a level of $350 \cdot 8$ (range 154.3-547.3) nmol/l within $2 \mathrm{~h}$ post-consumption, with a cumulative total $(0-$ $2 \mathrm{~h}$ ) serum concentration reaching 591.7 (range 197.3$986 \cdot 1) \mathrm{nmol} / \mathrm{l}$ over the $2 \mathrm{~h}$ sampling period (Table 2 ).

Magnification of the chromatograms revealed the appearance of many small peaks that were at concentrations too low to adequately identify but were well above any baseline noise. Adequate structural identification was not possible at this concentration but many peaks had spectral characteristics representative of anthocyanins ( $\lambda_{\max }$ (vis) in the $500 \mathrm{~nm}$ range). Analysis of the total peak areas at $525 \mathrm{~nm}$ (all integratable peaks with $\lambda_{\max }$ (vis) $>500 \mathrm{~nm}$ not observed in baseline samples) revealed that the urine total concentration of anthocyanins and/or 'anthocyanin-like' compounds reached levels as high as $22.7 \mu \mathrm{mol} / \mathrm{l}$ within $5 \mathrm{~h}$ post-consumption. The total $(0-2 \mathrm{~h})$ serum levels reached $997.5 \mathrm{nmol} / \mathrm{l}$ within $2 \mathrm{~h}$ post-consumption of the extract. (The concentration of unknown compounds was based on cyanidin 3-galactoside molar equivalents.) These results indicate that the body may have the capacity to transform anthocyanins into numerous metabolites, many of which probably go undetected as a result of their substantial numbers and subsequently low concentrations.

Efforts to identify the main anthocyanin metabolites through enzymic hydrolysis experiments were unsuccessful, as the enzymes $\beta$-galactosidase, $\beta$-glucuronidase, and $\beta$-glucosidase had affinities for all anthocyanin metabolite peaks to varying extents. This led us to suspect the presence of both glycoside and glucuronide derivatives of cyanidin in our samples. GC analysis, post-acid hydrolysis and derivativisation of sugars were also utilised to identify the possible glycosylating structures (galactose, glucose, arabinose, xylose, glucuronic acid). These results suggested that both glycosides and glucuronides were present. However, as a result of the low concentrations and large numbers of derivatised sugars produced, adequate chromatographic identification was not possible. Further analysis using electrospray ionisation-MS was necessary for sufficient identification of the potential metabolites.

Identification of urinary metabolites (Table 1) was based on the matching of molecular weights for parent and daughter fragments, along with the retention time, $\lambda_{\max }$ (vis) and 
$\mathrm{E}_{440}: \mathrm{E}_{\max }$, to that of available standards (Table 3 and Fig. 2). The data obtained indicated the presence of both cyanidin 3galactosides ( $\mathrm{m} / \mathrm{z} 449$; Fig. 1, peak 2$)$ and cyanidin glucuronides $(\mathrm{m} / \mathrm{z}, 463$; Fig. 1 , peak 3$)$. There are little data regarding the identification of anthocyanin glucuronides to date (Wu et al. 2002; Felgines et al. 2003). However, the urinary excretion of other flavonoid and isoflavonoid glucuronides such as catechin, quercetin, and genisten has been well documented (Wermeille et al. 1983; Hollman \& Katan, 1998; Piskula \& Terao, 1998; Holder et al. 1999; Okushio et al. 1999; Walle et al. 2000; Williamson et al. 2000; Oliveira et al. 2002).

Mono- and dimethylated cyanidin 3-galactoside derivatives $(\mathrm{m}+14$, peaks 4 and 5 , and $\mathrm{m}+28$, peak 9; Fig. 1$)$ and glucuronide derivatives $(\mathrm{m}+14$, peaks 6 and 7 , and $\mathrm{m}+28$, peak 10; Fig. 1) were also indicated in the present investigation (Table 1). Monomethylated derivatives had similar retention times and $\mathrm{E}_{440}: \mathrm{E}_{\max }$ to the peonidin 3 -glucoside standard. Only a few researchers have reported methylated anthocyanins in the urine and blood of human subjects and animals (Miyazawa et al. 1999; Tsuda et al. 1999). However, methylated derivatives of quercetin and catechin have been documented extensively (Harborne, 1958; Wermeille et al. 1983; Hollman \& Katan, 1998; Miyazawa et al. 1999; Okushio et al. 1999; Day \& Williamson, 2001; Donovan et al. 2001). In addition, oxidative modification was also indicated in one metabolite (peak $11 ; \mathrm{m} / \mathrm{z} 493)$ by an increased mass $(\mathrm{m}+16)$ above the di-methyl derivative of cyanidin 3-galactoside (represented by $\mathrm{m} / \mathrm{z}$ 477, peak 9; Fig. 1). This structure $(\mathrm{m} / \mathrm{z}$ 493/331) matched the molecular weights for parent and daughter fragments of malvidin 3-galactoside, had a similar retention time to that of the malvidin 3-glucoside standard but differed in spectral characteristics (Tables 1 and 3). No other oxidised derivatives of anthocyanins have been previously identified to our knowledge. Comparisons were made between anthocyanin metabolites in the serum and those in the urine; unfortunately, as a result of low concentrations of individual anthocyanins in the serum, the collection and concentration of a sufficient quantity of sample was not possible. Consequently, MS analysis using the above methods (p. 934) could not be utilised. However, retention times and UV-visible spectra were obtainable and the data indicated that the serum peaks matched the peaks identified in the urine (Table 2 and Fig. 1). When comparisons were made between urinary and serum metabolites, it was apparent that one anthocyanin metabolite (peak 11) occurred in the urine but not in the serum (Fig. 1, traces C and D). The identification of an anthocyanin metabolite exclusively in the urine may indicate that this metabolic product is either formed exclusively in the kidney, accumulates in the kidney, or the concentration of this metabolite in the serum may have been below the detection limit of our methodology. MS analysis (of the urinary metabolite) revealed this compound (peak 11) to be a highly metabolised cyanidin 3-galactoside (indicated by two methylations and one hydroxylation) having the same mass and daughter fragment as malvidin 3 -galactoside $(\mathrm{m} / \mathrm{z}$ 449/331). Tsuda et al. (1999) have previously reported the methylation of cyanidin 3-glucoside at both the $3^{\prime}$ and $4^{\prime}$ positions in the liver of rats. Also, the addition of hydroxyl groups to flavones has been characterised in animal models, where these metabolites were identified in the urine (Buset \& Scheline, 1980; Hollman \& Katan, 1998). In theory, dimethylation of the B-ring, resulting in reduced polarity, could merit further phase I cytochrome $\mathrm{P} 450$ oxidation, as observed with other flavonoids (Griffiths, 1982), in an attempt to increase the water solubility of the structure for elimination in the urine. This may explain the appearance of this compound in the urine while not in the serum. The parent aglycone cyanidin was not identified in any urine or serum samples. This result is consistent with other studies on anthocyanin metabolism as anthocyanin aglycones are generally regarded as unstable at physiological $\mathrm{pH}$ (Tsuda et al. 1999; Wu et al. 2002; Felgines et al. 2003).

Compounds having identical molecular weights but different retention times and absorption spectra were observed and are probably the result of methylation of the ortho hydroxyls of the cyanidin B-ring (i.e. $3^{\prime}$ v. $4^{\prime}$ methylation, peaks 4 and 5, 6 and 7; Fig. 1). Methylation at different sites of the B-ring may cause a slight shift in retention time, producing two distinct peaks on an HPLC chromatogram. It should be noted that the pathway we have proposed, in Fig. 3, only accounts for the derivatisation of the B-ring, which has been documented as a probable site for methylation and oxidation of flavonoids (Griffiths, 1982; Hollman \& Katan, 1998; Tsuda et al. 1999; Doostdar et al. 2000). However, methylation and hydroxylation at other sites of cyanidin may be possible and cannot be excluded using the methodologies used in the present study. In addition, the sites of anthocyanin glucuronide conjugation in man are unknown. The glucuronidation of quercetin, a flavonoid of similar structure to anthocyanins, has been documented at the $4^{\prime}, 3^{\prime}, 7$, and 3 positions of the polyphenol ring (Day et al. 2000). The absence of available standards for anthocyanin metabolites, along with the problems associated with compound identification when sample impurities exist, prevents the undeniable identification of the metabolites. NMR is required to elicit the actual positioning of the glucuronide, hydroxyl, or methyl subgroups.

Total urinary analysis of identified peaks revealed that cyanidin galactosides (cyanidin 3-galactoside and derivatives of cyanidin 3-galactoside) accounted for $84.0 \%$ (15.0 (range $11.5-18.7) \mu \mathrm{mol} / \mathrm{l}$ ) of the identified anthocyanins. Of these, $55.3 \%(9.9$ (range $7 \cdot 2-12.6) \mu \mathrm{mol} / \mathrm{l})$ was the parent compound cyanidin 3-galactoside (10.6\% glucuronides; 1.9 (range 1.5-2.4) $\mu \mathrm{mol} / \mathrm{l}$; Table 1). Accordingly, cyanidin galactosides accounted for $89.4 \%$ (529.3 (range 160.0-898.6) $\mathrm{nmol} / \mathrm{l}$ ) of the anthocyanins $(8.6 \%$ or 51.1 (range $30.8-71.4$ ) nmol glucuronides/1; Table 1) in the serum, with $66.0 \%$ (390.6 (range 119.4-661.9) nmol/l) being the parent compound cyanidin 3 -galactoside. The high percentage of anthocyanin glycosides in relation to glucuronides as observed in the present trial has also been reported by Wu et al. (2002) (90\% anthocyanin glycosides) but is not consistent with other flavonoid studies found in the literature. Most studies of flavonoid glycoside consumption indicate that the major metabolites in the urine are the glucuronide derivatives of the parent compound (Piskula \& Terao, 1998; Holder 
et al. 1999; Kuhnle et al. 2000; Donovan et al. 2001; Oliveira et al. 2002; Felgines et al. 2003). Felgines et al. (2003) recently reported that approximately $80 \%$ of excreted anthocyanins were monoglucuronides. The high percentage of glycoside observed in the present trial may be the result of the consumption of a high dose of anthocyanins and may not occur under more normal physiological concentrations.

Total analysis of identified peaks revealed that methyl derivatives of cyanidin galactosides and glucuronides accounted for 37 and $33 \%$ of the identified metabolites in the urine and serum, respectively. Although a high percentage of metabolites were methylated in the present investigation, the level of methylation, as well as oxidation, observed may have been, as suggested earlier, the result of the high dose of anthocyanins consumed.

We found no evidence of sulfation in the present study; however, studies indicate this pathway can be easily saturated (Dutton, 1980; Laitinen \& Watkins, 1986; Williamson et al. 2000; Oliveira et al. 2002). As the sulfation pathway is substrate limited, it is therefore possible that this pathway was overwhelmed by the high concentration of anthocyanin glycosides in the present investigation, and any sulfate derivatives appearing in the urine or blood may have been at a concentration below the detection limit of our methodology. It is also possible that metabolites of lower concentration had similar retention times (overlapping peaks on the chromatogram) to those of more concentrated metabolites and were not identified and collected. This illustration could also be used to explain why no arabinosides, glucosides, or xylosides were identified in the urine and serum. Alternatively, the route of absorption for anthocyanin glycosides may have specificity towards galactosides over, arabinosides or xylosides (Williamson et al. 2000).

Researchers have reported that many flavonoid glycosides are cleaved before entry into intestinal cells where they are either transported to the liver as aglycones, or metabolised to glucuronide and/or sulfide conjugates before transport (Hollman \& Katan, 1998; Spencer et al. 1999; Kuhnle et al. 2000; Williamson et al. 2000; Donovan et al. 2001; Oliveira et al. 2002). In the present investigation, both glycoside and glucuronides were identified in the urine and serum. This evidence leads us to believe that there may be more than one route of absorption. The combination of the present results and others in the literature seems to indicate that a dose-dependent or saturatable metabolic pathway may predominate (Mizuma et al. 1994; Hollman et al. 1995; Wolffram et al. 1995). This could explain the inconsistency in findings reported between studies using varying doses of anthocyanins and flavonoids.

The metabolism of anthocyanins (particularly by colonic microflora) may also result in the formation of phenolic acids, phenolic acid residues, $\mathrm{H}$, or $\mathrm{CO}_{2}$ (Rozman, 1986; Hollman \& Katan, 1998). Tsuda et al. (1999) reported that cyanidin 3-glycosides administered to rats were metabolised to protocatechuic acid. In the present investigation, protocatechuic acid was observed in trace amounts in both the urine ( $\mu \mathrm{g}$ concentrations) and serum (ng concentrations) samples; however, the source of the protocatechuic acid could not be determined and results have therefore been excluded from the present report. In addition, colonic microflora have significant potential to transform flavonoids into lower-molecular-weight compounds, which may also have protective biological activities. Future research in this area is essential to establish a more complete understanding of flavonoid metabolism.

To conclude, the present results indicate that orally administered cyanidin 3-glycosides are absorbed and are transported in human serum and urine as glycosides and glucuronides, both of which appear to undergo further methylation and oxidation. The contribution of each individual structure to the reported bioactivity (health effects) of anthocyanins should be the focus of future research.

\section{Acknowledgements}

The present study (Pacific Agri-Food Research Center contribution no. 2225) was supported by the National Sciences and Engineering Research Council of Canada, and the Ontario Ministry of Agriculture, Food and Rural Affairs.

\section{References}

Andriambeloson E, Magnier C, Haan-Archipoff G, Lobstein A, Anton R, Beretz A, Stoclet JC \& Andriantsitohaina R (1998) Natural dietary polyphenolic compounds cause endotheliumdependent vasorelaxation in rat thoracic aorta. J Nutr 128, 2324-2333.

Buset H \& Scheline RR (1980) Disposition of [2-14C]flavone in the rat. Acta Pharm Suec 17, 157-165.

Chandra A, Rana J \& Li Y (2001) Separation, identification, quantification and method validation of anthocyanins in botanical supplement raw material by HPLC and HPLC-MS. J Agric Food Chem 49, 3515-3521.

Day AJ, Bao Y, Morgan MRA \& Williamson G (2000) Conjugation position of quercetin glucuronides and effect on biological activity. Free Radic Biol Med 29, 1234-1243.

Day AJ \& Williamson G (2001) Biomarkers for exposure to dietary flavonoids: a review of the current evidence for identification of quercetin glycosides in plasma. $B r J$ Nutr 86, Suppl. 1, S105-S110.

Donovan JL, Crespy V, Manach C, Morand C, Besson C, Scalbert A \& Rémésy C (2001) Catechin is metabolised by both the small intestine and liver of rats. J Nutr 131, 1753-1757.

Doostdar H, Burke MD \& Mayer RT (2000) Bioflavonoids: selective substrates and inhibitors for cytochrome P450 CYP1A and CYP1B1. Toxicology 144, 31-38.

Dutton GJ (1980) Glucuronidation of Drugs and Other Compounds. Boca Raton, FL: CRC Press.

Felgines C, Talavera S, Gonthier MP, Texier O, Scalbert A, Lamaison JL \& Remesy C (2003) Strawberry anthocyanins are recovered in urine as glucuro- and sulfoconjugates in humans. J Nutr 133, 1296-1301.

Gao L \& Mazza G (1994) A rapid method for complete characterization of simple and acylated anthocyanins by high performance liquid chromatography and capillary gas liquid chromatography. J Agric Food Chem 42, 118-125.

Griffiths LA (1982) Mammalian metabolism of flavonoids. In The Flavonoids: Advances in Research, pp. 681-718 [T Mabry and J Harborne, editors]. London: Chapman \& Hall.

Harborne JB (1958) Spectral methods of characterizing anthocyanins. Biochem J 70, 22-28.

Holder CL, Churchwell MI \& Doerge DR (1999) Quantification 
of soy isoflavones, genistein and daidzein, and conjugates in rat blood using LC/ES-MS. J Agric Food Chem 47, 3764-3770.

Hollman PC, de Vries JH, van Leeuwen SD, Mengelers MJ \& Katan MB (1995) Absorption of dietary quercetin glycosides and quercetin in healthy ileostomy volunteers. Am $J$ Clin Nutr 62, 1276-1282.

Hollman PC \& Katan MB (1998) Absorption, metabolism and bioavailability of flavonoids. In Flavonoids in Health and Disease, pp. 483-522 [CA Rice-Evans and L Packer, editors]. New York: Marcel Dekker, Inc..

Kamei H, Kojima T, Hasegawa M, Koide T, Umeda T, Yukawa T \& Terabe K (1995) Suppression of tumor cell growth by anthocyanins in vitro. Cancer Invest 13, 590-594.

Kuhnle G, Spencer JP, Chowrimootoo G, Schroeter H, Debnam ES, Srai SK, Rice-Evans C \& Hahn U (2000) Resveratrol is absorbed in the small intestine as resveratrol glucuronide. Biochem Biophys Res Commun 272, 212-217.

Laitinen M \& Watkins JB (1986) Mucosal biotransformations. In Gastrointestinal Toxicology, pp. 169-192 [K Rozman and O Hänninen, editors]. New York: Elsevier.

Laplaud PM, Lelubre A \& Chapman MJ (1997) Antioxidant action of Vaccinium myrtillus extract on human low density lipoproteins in vitro: initial observations. Fundam Clin Pharmacol 11, 35-40.

Mazza G (2000) Health aspects of natural colors. In Natural Food Colorants: Science and Technology, pp. 289-314 [GJ Lauro and FJ Francis, editors]. New York: Marcel Dekker, Inc.

Mazza G, Kay C, Cottrell T \& Holub B (2002) Absorption of anthocyanins from blueberries and serum antioxidant status in humans. J Agric Food Chem 50, 7731-7737.

Miyazawa T, Nakagawa K, Kudo M, Muraishi K \& Someya K (1999) Direct intestinal absorption of red fruit anthocyanins, cyanidin-3-glucoside and cyanidin-3,5-diglucoside, into rats and humans. J Agric Food Chem 47, 1083-1091.

Mizuma T, Ohta K \& Awazu S (1994) The beta-anomeric and glucose preferences of glucose transport carrier for intestinal active absorption of monosaccharide conjugates. Biochim Biophys Acta 1200, 117-122.

Mülleder U, Murkovic M \& Pfannhauser W (2002) Urinary excretion of cyanidin glycosides. J Biochem Biophys Methods 53, 61-66.

Murkovic M, Adam U \& Pfannhauser W (2000) Analysis of anthocyane glycosides in human serum. Fresenius $J$ Anal Chem 366, 379-381.

Okushio K, Suzuki M, Matsumoto N, Nanjo F \& Hara Y (1999)
Identification of (-)-epicatechin metabolites and their metabolic fate in the rat. Drug Metab Dispos 27, 309-316.

Oliveira EJ, Watson DG \& Grant MH (2002) Metabolism of quercetin and kaempferol by rat hepatocytes and the identification of flavonoid glycosides in human plasma. Xenobiotica 32, 279-287.

Parthasarathy S, Khan-Merchant N, Penumetcha M \& Santanam N (2001) Oxidative stress in cardiovascular disease. $J$ Nucl Cardiol 8, 379-389.

Piskula MK \& Terao J (1998) Accumulation of (-)-epicatechin metabolites in rat plasma after oral administration and distribution of conjugation enzymes in rat tissues. J Nutr 128, $1172-1178$

Rozman K (1986) Fecal excretion of toxic substances. In Gastrointestinal Toxicology, pp. 119-145 [K Rozman and O Hänninen, editors]. New York: Elsevier.

Spencer JP, Chowrimootoo G, Choudhury R, Debnam ES, Srai SK \& Rice-Evans C (1999) The small intestine can both absorb and glucuronidate luminal flavonoids. FEBS Lett $\mathbf{4 5 8}$, 224-230.

Suda I, Oki T, Masuda M, Nishiba Y, Furuta S, Matsugano K, Sugita K \& Terahara N (2002) Direct absorption of acylated anthocyanin in purple-fleshed sweet potato in rats. J Agric Food Chem 50, 1672-1676.

Trevithick JR \& Mitton KP (1999) Antioxidants and diseases of the eye. In Antioxidant Status, Diet, Nutrition, and Health, pp. 545-566 [AM Papus, editor]. New York: CRC Press.

Tsuda T, Horio F \& Osawa T (1999) Absorption and metabolism of cyanidin 3-O-beta-D-glucoside in rats. FEBS Lett 449, $179-182$.

Walle T, Otake Y, Walle UK \& Wilson FA (2000) Quercetin glucosides are completely hydrolyzed in ileostomy patients before absorption. J Nutr 130, 2658-2661.

Wermeille M, Turin E \& Griffiths LA (1983) Identification of the major urinary metabolites of (+)-catechin and 3-O- methyl$(+)$-catechin in man. Eur J Drug Metab Pharmacokinet 8, $77-84$.

Williamson G, Day AJ, Plumb GW \& Couteau D (2000) Human metabolic pathways of dietary flavonoids and cinnamates. Biochem Soc Trans 28, 16-22.

Wolffram S, Weber T, Grenacher B \& Scharrer E (1995) A $\mathrm{Na}(+)$-dependent mechanism is involved in mucosal uptake of cinnamic acid across the jejunal brush border in rats. J Nutr 125, 1300-1308.

Wu X, Cao G \& Prior RL (2002) Absorption and metabolism of anthocyanins in elderly women after consumption of elderberry or blueberry. J Nutr 132, 1865-1871. 